\title{
Salicylates increase insulin levels rather than insulin actions
}

An increase in serum insulin levels rather than insulin action mediates the glucoselowering effects of low-dose salicylates, according to a randomized, placebo-controlled trial. Beneficial effects of salicylates on fasting and postprandial glucose levels are well known, but previous studies reported conflicting hypotheses for their mechanisms of action.

Salicylates are commonly used to treat rheumatic pain and have an established safety profile. As chronic, subclinical inflammation is often associated with obesity and increases the risks of obesityrelated insulin resistance and type 2 diabetes mellitus, researchers suggest that salicylates could prevent or ameliorate obesity-related diabetes mellitus.

Koska and colleagues analyzed data obtained from 40 nondiabetic patients (aged 18-45 years) with BMI $\geq 30 \mathrm{~kg} /$ $\mathrm{m}^{2}$ who were randomly allocated $3 \mathrm{~g}$ salsalate (a dimer of salicylic acid) or placebo daily for 7 days. After this time, salsalate treatment significantly decreased fasting glucose levels and glucose area under the curve versus placebo during an oral glucose-tolerance test. Furthermore, an apparently significant increase in the rate of glucose disposal in the salsalate group was observed, as measured by a euglycemic-hyperinsulinemic clamp. This increase disappeared, however, when values were adjusted for the increased insulin concentrations during the clamp.

These findings show that salicylates are promising candidates for hyperglycemia treatment in obese patients, but more data are needed to understand how they increase insulin levels.

Julianna Vig

Original article Koska, J. et al. The effect of salsalate on insulin action and glucose tolerance in obese nondiabetic patients: results of a randomized double-blind placebo-controlled study. Diabetologia 52, 385-393 (2009). 